\title{
VARIABILIDADE ESPACIAL DA TAXA DE INFILTRAÇÃO DE ÁGUA E DA ESPESSURA DO HORIZONTE A, EM UM ARGISSOLO VERMELHO-AMARELO, SOB DIFERENTES USOS ${ }^{(\mathbf{1})}$
}

\author{
F. C. BERTOLANI ${ }^{(2)} \&$ S. R. VIEIRA(3)
}

\begin{abstract}
RESUMO
O experimento foi realizado no município de Vera Cruz (SP), no período de 1996 a 1998, com vistas em estudar a variabilidade espacial da taxa de infiltração de água em solo saturado e a espessura do horizonte $A$. $O$ solo utilizado foi um Argissolo Vermelho-Amarelo eutrófico abrúptico, apresentando evidentes sinais de processos erosivos. As determi nações de infiltração nos horizontes A, E e Bt, bem como da espessura do horizonte $A$, foram obtidas em malhas regulares de $\mathbf{4 0} \times \mathbf{4 0} \mathrm{m}$, com espaçamento de $5 \times 5 \mathrm{~m}$, totalizando 64 pontos, em café, pastagem e mata/capoei ra. A análise das medi das de disper são estatística revelou elevada variação das taxas de infiltração, bem como da espessura do horizonte A. Os semivariogramas indicaram a existência de dependência espacial da infiltração no horizonte $E$, em todos os usos amostrados. Com a utilização do semivariograma cruzado, detectou-se a existência de correlação espacial entre a espessura do horizonte A e a infiltração, em cultivo de café e na mata/capoeira. A cobertura vegetal existente na mata/capoeira reduziu a perda do horizonte $A$, o que foi comprovado pela maior espessura e menor variação espacial.
\end{abstract}

Termos de indexação: permeâmetro, geoestatística, semivariograma, áreas degradadas.

\footnotetext{
(1) Parte da Tese de Mestrado do primeiro autor, apresentada à Faculdade de Engenharia Agrícola, Universidade de Campinas UNICAMP. Recebido para publicação em junho de 2000 e aprovada em junho de 2001.

(2) Pós-graduando do Departamento de Água e Solo, Faculdade de Engenharia Agrícola, Universidade de Campinas - FEAGRI/ UNICAMP. Caixa Postal 1170, CEP 13083-970 Campinas (SP). Bolsista da FAPESP.

(3) Pesquisador Científico no Centro de Sol os e Recursos Agroambientais, Instituto agronômico de Campinas - IAC. Caixa Postal 28, CEP 13001-970 Campinas (SP). Bolsista do CNPq.
} 


\title{
SUMMARY: SPATIAL VARIABILITY OF SOIL WATER INFILTRATION RATE AND A HORIZON THICKNESS IN A RED-YELLOW PODZOLIC SOIL UNDER DIFFERENT USES
}

\begin{abstract}
The spatial variability of soil water infiltration rate and A horizon thickness were studied in a Red-Yellow Podzolic soil (Kandudalf) showing erosion signs, located in Vera Cruz, SP. Field measurements weretaken in regular 5 meter spacing squaregrid, resulting in 64 sampling points wheretheinfiltration rateat the A, E and B horizon, and A horizon thickness were determined in coffee, pasture and natural vegetation. Statistical analysis showed high variability in the soil water infiltration rate and in the A horizon thickness. A semivariogram showed the existence of spatial dependence on infiltration rate in the $E$ horizon in all land uses. A cross-semivariogram showed spatial correlation between A horizon soil thickness and soil water infiltration ratein coffeecrop and natural vegetation. The natural vegetation cover protected A horizon soil loss by erosion, as shown by its greater thickness and lesser spatial variation.
\end{abstract}

Index terms: permeameter, geoestatistics, semivariogram, degraded areas.

\section{INTRODUÇÃO}

A agricultura moderna deve basear-se na sustentabilidade dos recursos naturais, bem como na otimização do uso do solo, sem causar degradação do meio ambiente. Neste sentido, métodos que contribuem para a mel horia da qualidade dos solos favorecem a prática de uma agricultura mais equilibrada.

O conhecimento do comportamento físico do solo possi bilita adequar as atividades agrícol as à aptidão do solo, o que pode reduzir a erosão das camadas superficiais e, por conseqüência, sua degradação física, química e biológica. A infiltração de água no solo e a espessura do horizonte $A$ fornecem indicativos da qualidade do solo, gerando informações com as quais é possível verificar a existência de camadas compactadas, encrostamento superficial, bem como as conseqüências dos processos erosivos.

A variabilidade espacial da taxa de infiltração e da espessura do horizonte A é atribuída não apenas aos processos de formação do sol o, como também aos seus sistemas de preparo, os quais modificam a rugosidade superficial ea porosidade do solo (Hillel, 1980; Paz \& Taboada, 1996). Essas alterações provocam reduções nas taxas de infiltração e, conseqüentemente, perda de solo da camada superficial decorrentedo escorrimento superficial da água.

Em sistemas de cultivos altamente tecnificados, é fundamental ter o conhecimento da variabilidade espacial destes atributos, o que poderá contribuir para a redução de custos nos sistemas de produção. Albuquerque et al. (1996), utilizando técnicas de geoestatística, encontraram correlação positiva entre a espessura do horizonte A e a produtividade do milho em área de plantio direto. Por outro lado, um resultado como este depende da escala amostrada, da posição na encosta e, principalmente, do tipo de solo.

Os atributos do solo, após sofrerem sucessivas alterações provocadas pelas atividades agrícolas e, conseqüentemente, pelos processos erosivos, comportam-se de forma bastante diferenciada ao Iongo da paisagem. Consi derando essas variações e a dependência espacial existente nos atributos do solo, a utilização de amostragem aleatória seria insuficiente para representar toda uma classe de solo, incorrendo em sérios erros na tomada de decisões realizadas com base nessas informações (Vieira et al., 1983; Prevedello, 1987).

A variabilidadeespacial, portanto, deveser levada em consideração, tanto em experimentos científicos, como em áreas destinadas à exploração comercial, para não incorrer em erros de planejamentos.

Dentro desse contexto, o presente trabal ho teve como objetivo determinar a variabilidade espacial da infiltração de água em solo saturado e da espessura do horizonte $A$, nos usos decafé, pastagem emata/capoeira, em um Argissol oVermel ho-Amarelo eutrófico abrúptico com evidentes sinais de processos erosivos.

\section{MATERIAIS E MÉTODOS}

O município de Vera Cruz situa-se no oeste do estado de São Paulo, especificamente no sudoeste do seu Planal to Ocidental. Segundo classificação de Köppen, o clima na área estudada apresenta uma estação chuvosa compreendida entre outubro e 
março, com preci pitação pl uviométrica e temperatura média anual, respectivamente, de 1.030 mm e 22,5$24,5^{\circ} \mathrm{C}$. J á com a estação seca, compreendida entre abril e setembro, os referidos parâmetros climáticos são de $333 \mathrm{~mm}$ e $18-22^{\circ} \mathrm{C}$ (dados fornecidos pelo Centro de Ecofisiologia e Biofísica - IAC).

O trabalho foi realizado, no período de 1996 a 1998, em um Argissolo Vermel ho-Amarel o eutrófico abrúptico (EMBRAPA, 1999), que é a classe de solo predominantenessa região (Bertolani et al., 2000a), tendo, como material de origem, o arenito do Grupo Bauru, Formações Marília eAdamantina (IPT, 1986). Nesta classe de solo, a seqüência de horizontes A, E e Bt mostra transição abrupta do teor de argila, do horizonte $\mathrm{E}$ para o $\mathrm{Bt}$, o que Ihe confere alta susceptibilidade à erosão. Dado esse aspecto, juntamentecoma utilização de manejos inadequados, o solo estudado apresenta-se, nessa região, em avançado estádio de degradação (Bertolani et al., 2000a).

Para determinar a espessura do horizonte $A$ (Esp-A) e as taxas de infiltração de água nos horizontes A (Inf-A), E (Inf-E) e Bt (Inf-B), utilizaram-se parcelas quadriculadas (mal has de amostragem) de $40 \times 40 \mathrm{~m}$, com pontos espaçados de 5 em $5 \mathrm{~m}$, totalizando 64 pontos. Essas malhas de amostragem foram instaladas nos usos de café, pastagem e na mata/capoeira, predominantes na área estudada. As determinações das taxas de infiltração de água do horizonte $\mathrm{E}$ foram realizadas próximo ao horizonte diagnóstico de subsuperfície (Bt), com o objetivo de detectar a influência da transição textural abrupta.

Os valores da espessura do horizonte A foram obtidos por meio de medições com régua graduada em milímetros, nos mesmos pontos de amostragem das mal has quadriculadas utilizadas na determinação da infiltração. Considerou-se a espessura do horizonte A como sendo o somatório dos horizontes superficiais arenosos (A e E) que estão acima da transição textural abrupta, levando em conta os intensos processos erosivos existentes na área, os quais provocaram a remoção parcial ou completa do horizonte $A$, restando em determinados Iocais apenas o horizonte $\mathrm{E}$.

Para obter os mapas tridimensionais da espessura do horizonte A, utilizou-se o progama SURFER (Keckler, 1995), tendo sido os dados interpolados por meio da técnica da krigagem, a qual utiliza os parâmetros do semivariograma (Krige, 1951).

A taxa deinfiltração deágua foi medida utilizando o permeâmetro de carga constante - modelo IAC efoi calculada usando-se a equação (Reynolds \& Elrick, 1985):

$$
I=60\left[\frac{D p^{2}}{D o^{2}+4 D o H}\right] Q
$$

em que I é a taxa de infiltração de água em solo saturado $(\mathrm{mm} / \mathrm{h})$; Dp é o diâmetro do permeâmetro (cm); Do éo diâmetro do orifício da amostragem $(\mathrm{cm})$; $\mathrm{H}$ é a carga hidráulica utilizada na medição $(\mathrm{cm})$ e Q é o fluxo ( $\mathrm{mm} / \mathrm{min}$.) determinado na saturação.

Com relação à estatística descritiva, foram calculados os parâmetros: média, desvio-padrão, coeficiente de variação, mínimo, máximo, simetria e curtose (Snedecor, 1967), e, para a definição das classes de coeficiente de variação, utilizou-se a classificação de Pimentel Gomes (1984). A geoestatística foi utilizada com o propósito de avaliar a estrutura espacial dos atributos. O semi variograma expressa o grau de dependência espacial entre amostras dentro de um campo específico, sendo calculado pela expressão (Vieira et al., 1983):

$$
\gamma(\mathrm{h})=\frac{1}{2 \mathrm{~N}(\mathrm{~h})} \sum_{\mathrm{i}=1}^{\mathrm{N}(\mathrm{h})}\left[\mathrm{Z}\left(\mathrm{x}_{\mathrm{i}}\right)-\mathrm{Z}\left(\mathrm{x}_{\mathrm{i}+\mathrm{h}}\right)\right]^{2}
$$

em que $\gamma(h)$ é a semivariância; $N(h)$ é o número de pares de amostras, " $Z\left(x_{i}\right), Z\left(x_{i}+h\right)$ ", separadas por uma distância $h$; e $Z$ representa os val ores medidos dos atributos do solo. O gráfico de $\gamma(\mathrm{h})$ versus $h$ representa o semivariograma. O ajustematemático dos dados no gráfico define os seguintes parâmetros: 1 - efeito pepita (Co) - valor de $\gamma$ quando $h=0 ; 2$ alcance da dependência espacial (a) -éo valor deh quando a semivariância $(\gamma)$ estabiliza próximo a um valor constante; 3 - patamar (C) - valor da semivariância $(\gamma)$ quando $\mathrm{h}=\mathrm{a}$.

Os valores de semivariância obtidos por meio da equação [2] foram escalonados para facilitar a análise dos dados. Isto permitiu comparar os semivariogramas de diferentes variáveis, ou da mesma variável em diferentes tratamentos (Vieira et al., 1997). O exame dos parâmetros dos semivariogramas permitiu determinar o comportamento da dependência espacial e até que distância as propriedades mostravam tal dependência.

Para o ajuste dos modelos matemáticos aos semivariogramas, utilizou-se o método de val idação J ack-knifing, no qual foram analisados os valores de média e variância dos erros reduzidos (Souza et al., 1997), tendo sido considerados os modelos: esférico (Esf), exponencial (Esp) e gaussiano (Gau). Deacordo com vários autores (McBratney \& Webster, 1986; Cambardella et. al., 1994; Vieira et al., 1997; Bertolani et al.,2000b), tais model os são os utilizados, com maior freqüência, para avaliar os atributos do solo.

O semivariograma cruzado permitiu verificar a correlação espacial entre a infiltração de água no horizonte $\mathrm{A}$ e a sua espessura, nos diferentes usos estudados. Para o seu cálculo, utilizou-sea expressão (Vaudin et al., 1983):

$$
\gamma_{12}(h)=\frac{1}{2 N(h)} \sum_{i=1}^{N(h)}\left[Z_{1}\left(x_{i}\right)-Z_{1}\left(x_{i+h}\right)\right]\left[Z_{2}\left(x_{i}\right)-Z_{2}\left(x_{i+h}\right)\right]
$$


em que $Z_{1}$ representa a infiltração da água em solo saturado $\mathrm{e} Z_{2}$ a espessura do horizonte $A$.

A análise do semivariograma edo semivariograma cruzado, considerando o solo e respectivo uso, possibilitou não só verificar a variabilidade espacial da espessura do horizonte A e da infiltração deágua, nos diferentes usos, mas também correlacionar a estrutura de variação desses atributos. Isto pode fornecer indicativos da qualidade do solo, pois tal análise permite visualizar como estão ocorrendo as suas variações de acordo com as diferentes culturas (Albuquerque et al., 1996).

\section{RESULTADOS E DISCUSSÃO}

No município deVera Cruz, oArgissoloVermel hoAmarel o eutrófico abrúptico constitui-se em um solo com muitos problemas físicos, pois, além da textura contrastante entre os horizontes E e Bt, encontrase em rel evo ondulado a forte ondulado e apresenta horizontes arenosos acima da transição textural abrúptica (Bertolani et al., 2000a), estando, portanto, sujeito à formação de enxurradas freqüentes e, posteriormente, aos processos erosivos.

A variação de espessura do horizonte $A$ é um indicativo de erosão nessa classe de sol o, pois, quanto menos espesso for o horizonte superficial, mais rápida será a sua saturação hídrica por ocasião da ocorrência de chuvas intensas, aumentando a enxurrada. A saturação hídrica decorre principalmente do aumento do teor de argila em profundidade e da conseqüente diminuição da macropor osidade no horizonte Bt, reduzindo, assim, a velocidade de infiltração nesse horizonte (Bertolani, 1998). Esse fato pode ser notado nos dados de espessura do horizonte A (Figura 1), em café e pastagem, os quais apresentaram elevada variação, o que evidencia a ocorrência de processos erosivos com a retirada da vegetação natural.

Os valores da espessura no horizonte A, nos cultivos de café e pastagem, foram menores do que os observados para mata/capoeira. Tal resultado confirmou a importância da cobertura vegetal sobre a manutenção do horizonte superficial, em sol os que apresentam transição textural abrupta e com baixos teores de argila em superfície. Portanto, o sistema radicular e a cobertura vegetal, no horizonte superficial, aumentam a infiltração de água, reduzem o escorrimento superficial da água e, conseqüentemente, a perda de solo (Figura 1).

Utilizando a classificação de Pimentel Gomes (1984), verificaram-se valores de coeficiente de variação baixos a médios, para a espessura do horizonte A, eval ores al tos a muito altos, para a taxa de infiltração (Quadro 1). Contudo, esta el evada variação dos dados não provocou necessariamente dependência espacial, o que foi confirmado pelo

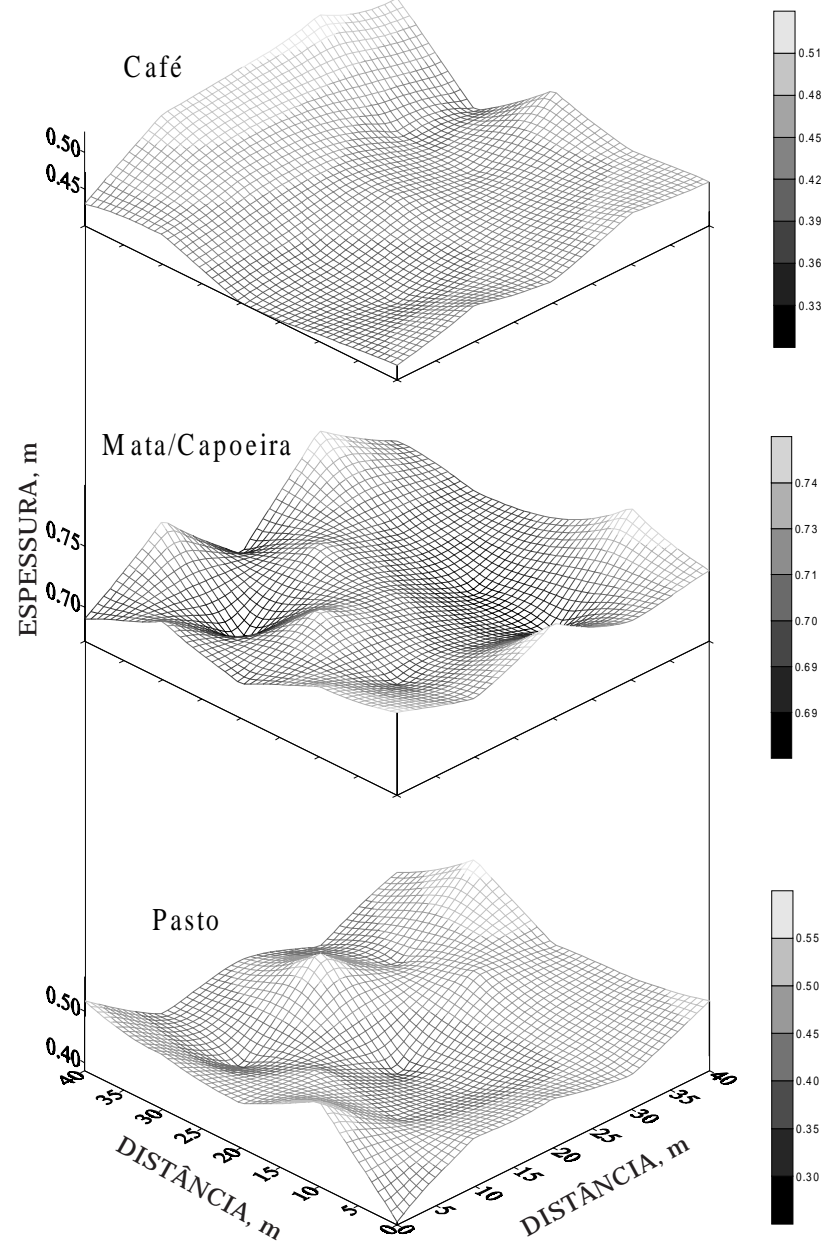

Figura 1. Mapa tridimensional da espessura do horizonte A, nos cultivos de café e pastagem e na mata/capoeira.

coeficiente de variação da infiltração no horizonte A, em mata/capoeira e pastagem, com valores médios e muito al tos, respectivamente, eapresentando efeito pepita puro (Quadro 2).

O efeito pepita puro pode-se referir a um erro analítico (variabilidade não explicada) ou pode ser devido a variações não detectadas, conforme a distância deamostragem utilizada (Cambardella et al., 1994). No entanto, a degradação física equímica do solo, como resultado de uso intensivo e processos erosivos, pode causar el evadas alterações na estrutura de dependência espacial, de tal modo que as variaç̃̃es dos atributos do sol o ocorram de forma aleatória. Resultados semel hantes também foram encontrados por outros autores eem sol os diferentes (Salviano, 1996; Berg \& Klamt, 1997).

O alcance da autocorrelação espacial, ou seja, a distância máxima na qual as variáveis estão espacialmente correlacionadas, foi comparado para os diferentes usos do sol o existentena área estudada. 
Quadro 1. Parâmetros da estatística descritiva utilizados nas malhas de amostragem para a infiltração de água nos horizontes A (Inf-A), E (Inf-E) e B (Inf-B) e espessura do horizonte A (Esp-A)

\begin{tabular}{|c|c|c|c|c|c|c|c|c|}
\hline Propriedade & Média & Variância & Desvio-padrão & CV & Mínimo & Máximo & Simetria & Curtose \\
\hline \multicolumn{9}{|c|}{ Café } \\
\hline Inf-A, mm/h & 217,9 & $6.964,0$ & 83,4 & 38,3 & 100,7 & 402,9 & 0,4 & 2,2 \\
\hline Inf-E, mm/h & 70,1 & $1.485,0$ & 38,5 & 54,9 & 21,5 & 144,5 & 0,4 & 1,7 \\
\hline Inf-B, mm/h & 20,5 & 81,6 & 9,0 & 43,9 & 5,3 & 43,1 & 0,5 & 2,7 \\
\hline Esp-A, cm & 44,6 & 12,6 & 3,5 & 7,9 & 40,0 & 53,0 & 0,6 & 2,6 \\
\hline \multicolumn{9}{|c|}{ Capoeira } \\
\hline Inf-A, mm/h & 407,5 & $6.581,0$ & 81,1 & 19,9 & 230,2 & 575,5 & $-0,3$ & 2,8 \\
\hline Inf-E, mm/h & 133,8 & $7.504,0$ & 86,6 & 64,7 & 50,4 & 474,8 & 2,4 & 10,2 \\
\hline Inf-B, mm/h & 50,9 & 284,0 & 16,8 & 33,1 & 21,5 & 86,3 & 0,2 & 2,3 \\
\hline Esp-A, cm & 71,3 & 5,2 & 2,3 & 3,2 & 67,0 & 75,0 & $-0,1$ & 2,2 \\
\hline \multicolumn{9}{|c|}{ Pasto } \\
\hline Inf-A, mm/h & 426,5 & $20.800,0$ & 144,1 & 33,8 & 215,8 & 805,7 & 0,9 & 3,3 \\
\hline $\mathrm{Inf}-\mathrm{E}, \mathrm{mm} / \mathrm{h}$ & 236,2 & $23.000,0$ & 151,7 & 64,2 & 48,5 & 567,1 & 0,8 & 2,6 \\
\hline Inf-B, mm/h & 48,1 & 730,4 & 27,0 & 56,2 & 13,6 & 103,2 & 0,6 & 2,4 \\
\hline Esp-A, cm & 46,6 & 24,6 & 4,9 & 10,6 & 38,0 & 57,0 & 0,1 & 2,4 \\
\hline
\end{tabular}

Quadro 2. Parâmetros do semivariograma escalonado para infiltração de água nos horizontes A (Inf-A), E (Inf-E) e B (Inf-B) e espessura do horizonte A (E sp-A)

\begin{tabular}{|c|c|c|c|c|c|c|}
\hline Propriedade & $\mathrm{Co}^{(1)}$ & $\mathbf{C 1}^{(2)}$ & $a^{(3)}$ & F S(4) & $M^{(5)}$ & $V^{(6)}$ \\
\hline & \multicolumn{6}{|c|}{ Café } \\
\hline Inf-A & 0,15 & 0,9 & 24 & 7.504 & 0,046 & 1,219 \\
\hline Inf-E & 0,2 & 1,1 & 22 & 248 & 0,217 & 0,936 \\
\hline Inf-B & \multicolumn{6}{|c|}{ Efeito pepita puro } \\
\hline Esp-A & \multicolumn{6}{|c|}{ E feito pepita puro } \\
\hline & \multicolumn{6}{|c|}{ Mata/capoeira } \\
\hline Inf-A & \multicolumn{6}{|c|}{ E feito pepita puro } \\
\hline Inf-E & 0,2 & 0,77 & 32 & 6.964 & 0,257 & 0,964 \\
\hline Inf-B & 0,25 & 0,95 & 31 & 1.485 & 0,311 & 1,023 \\
\hline Esp-A & \multicolumn{6}{|c|}{ Efeito pepita puro } \\
\hline & \multicolumn{6}{|c|}{ Pasto } \\
\hline Inf-A & \multicolumn{6}{|c|}{ Efeito pepita puro } \\
\hline Inf-E & 0,1 & 0,95 & 28 & 23.000 & 0,157 & 0,993 \\
\hline Inf-B & \multicolumn{6}{|c|}{ Efeito pepita puro } \\
\hline Esp-A & \multicolumn{6}{|c|}{ Efeito pepita puro } \\
\hline
\end{tabular}

${ }^{(1)}$ Efeito pepita. ${ }^{(2)}$ (C-Co). ${ }^{(3)}$ Alcance dos modelos ajustados. ${ }^{(4)}$ Fator de escalonamento. ${ }^{(5)}$ Média dos erros reduzidos. ${ }^{(6)}$ Variância dos erros reduzidos.

A dimensão deste parâmetro indica um raio dentro do qual os valores apresentam-se com grande semel hança esão correlacionados uns com os outros. Nota-se, no, quadro 2, que a maioria dos valores do alcance, encontrados nos diferentes usos, está em torno de $25 \mathrm{~m}$. Souza et al. (1997) sugerem, para futuras amostragens, que o interval o entre amostras tenha val ores menores que o alcance da dependência espacial. Portanto, quando houver a necessidade de mai or detalhamento dos atributos dos solos, para as condições estudadas, recomenda-se utilizar interval os menores que $25 \mathrm{~m}$. 
Comparando os semivariogramas escalonados (Figuras 2, 3 e 4), observou-se que a variabilidade da taxa de infiltração de água nos horizontes A e E foi semel hantenas mal has deamostragens contidas na mata/capoeira e na pastagem. Em ambas as malhas, observaram-se o efeito pepita puro, no horizonte A, e o mesmo valor de efeito pepita no horizonte $\mathrm{E}$ (Quadro 2). I sso pode indicar que o comportamento da infiltração deágua nos horizontes A e E, sob pastagem, assemel ha-se ao dos sol os em condições naturais.

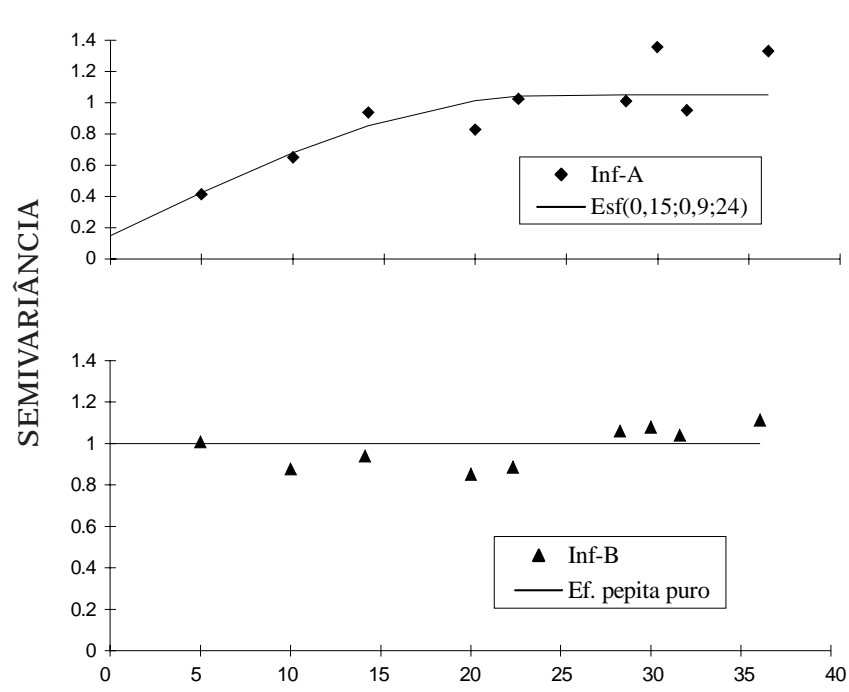

Por outro lado, esse mesmo comportamento não foi verificado para a cultura de café. Nesse caso, a amostragem regionalizada e a aplicação da geoestatística permitiram correlacionar atributos do solo e, conseqüentemente, solucionar problemas ligados à inter pretação e posterior uso desses dados (Vieira et al., 1981). Os parâmetros dos semivariogramas indicaram também, para a espessura do horizonte $A$, a ocorrência de dependência espacial apenas no cultivo de café (Figura 2). Nos demais usos, verificou-se o efeito

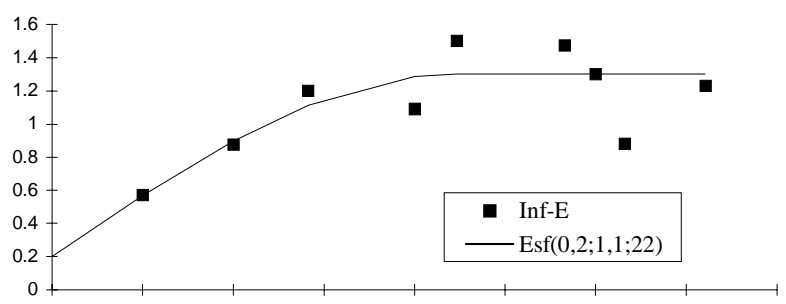

Figura 2. Semivariogramas escalonados da infiltração de água nos horizontes A, E e Bt e da espessura do horizonte A, sob o cultivo de café.
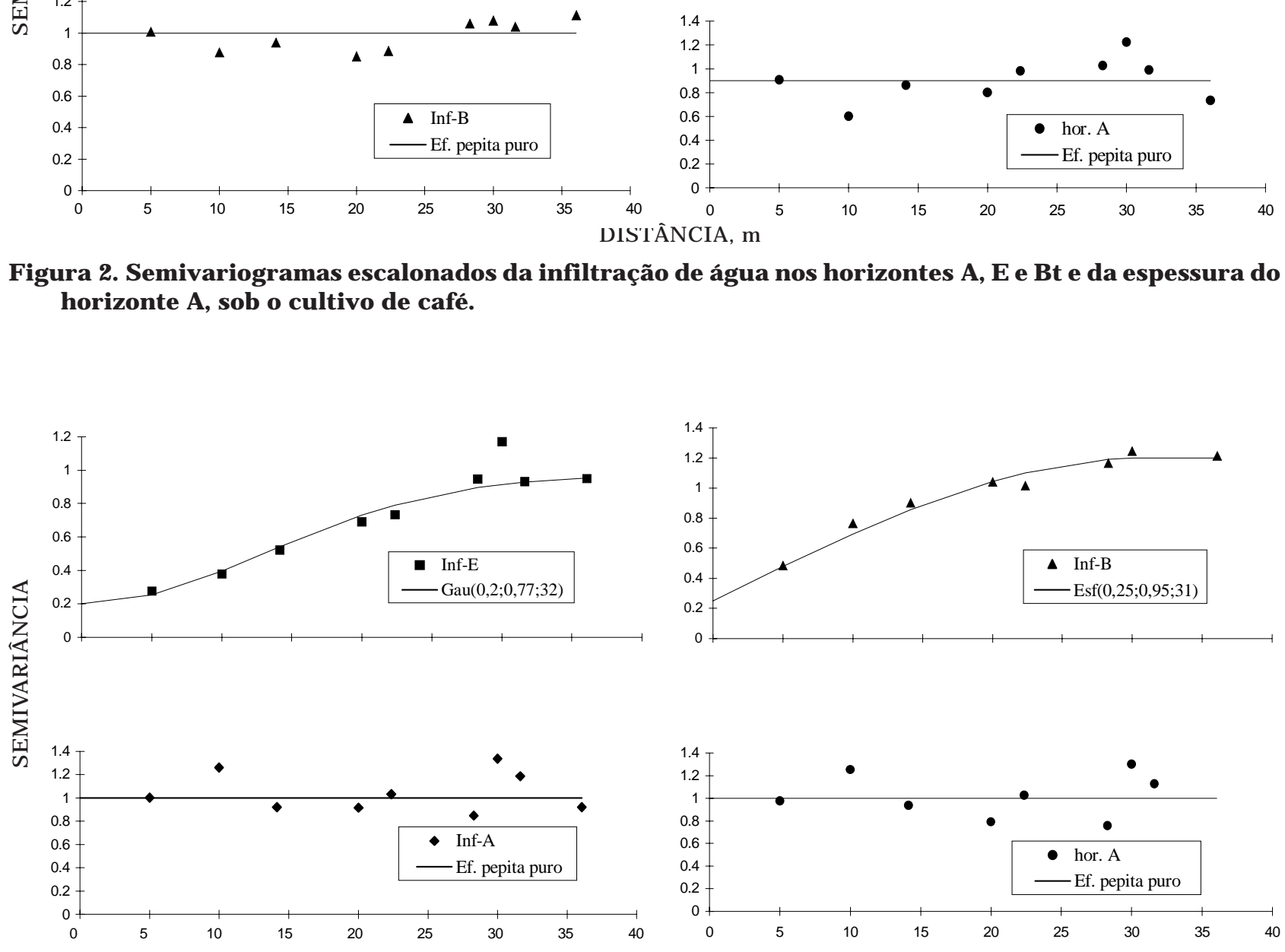

Figura 3. Semivariogramas escalonados da infiltração de água no horizonte A, E e Bt e da espessura do horizonte A, sob mata/capoeira. 


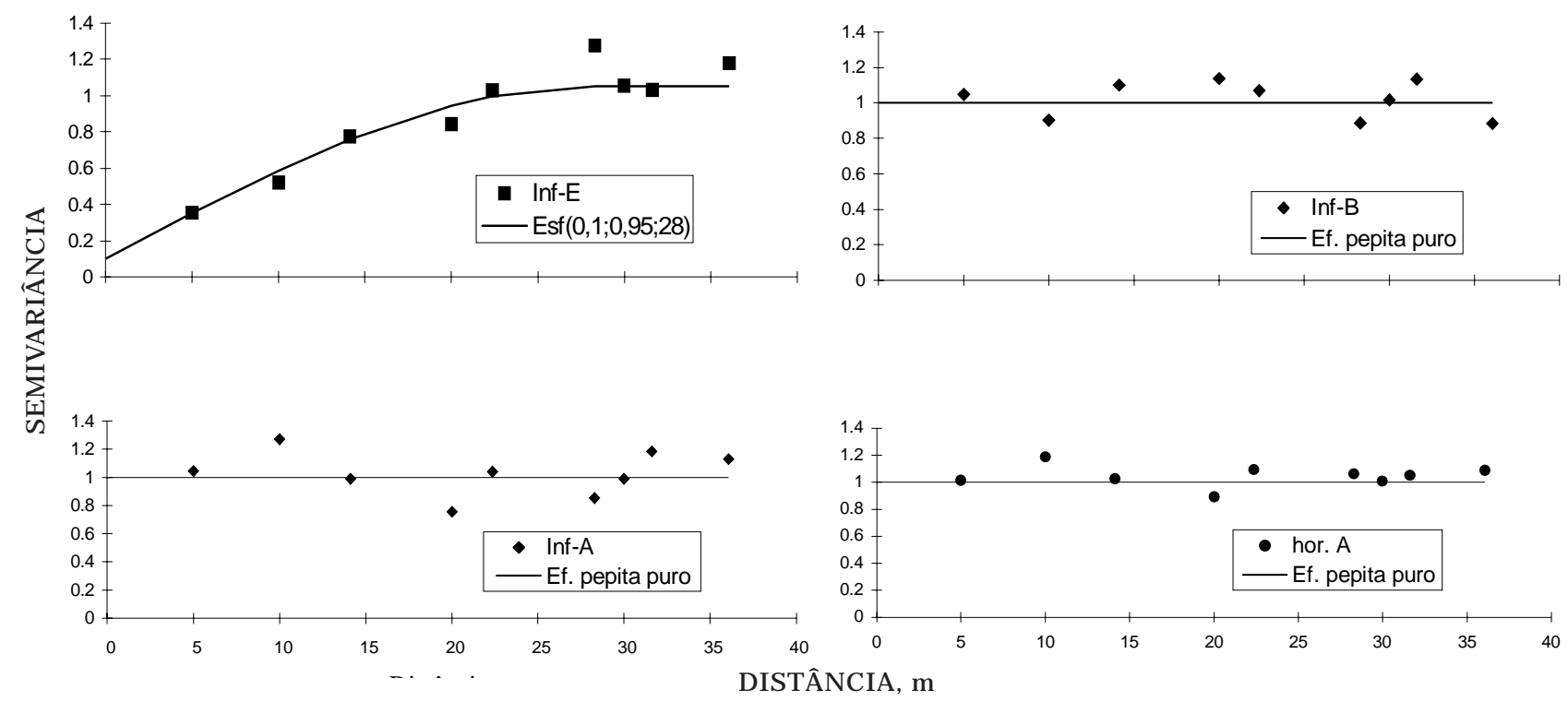

Figura 4. Semivariogramas escalonados da infiltração de água nos horizontes A, E e Bt e da espessura do horizonte A, sob pastagem.

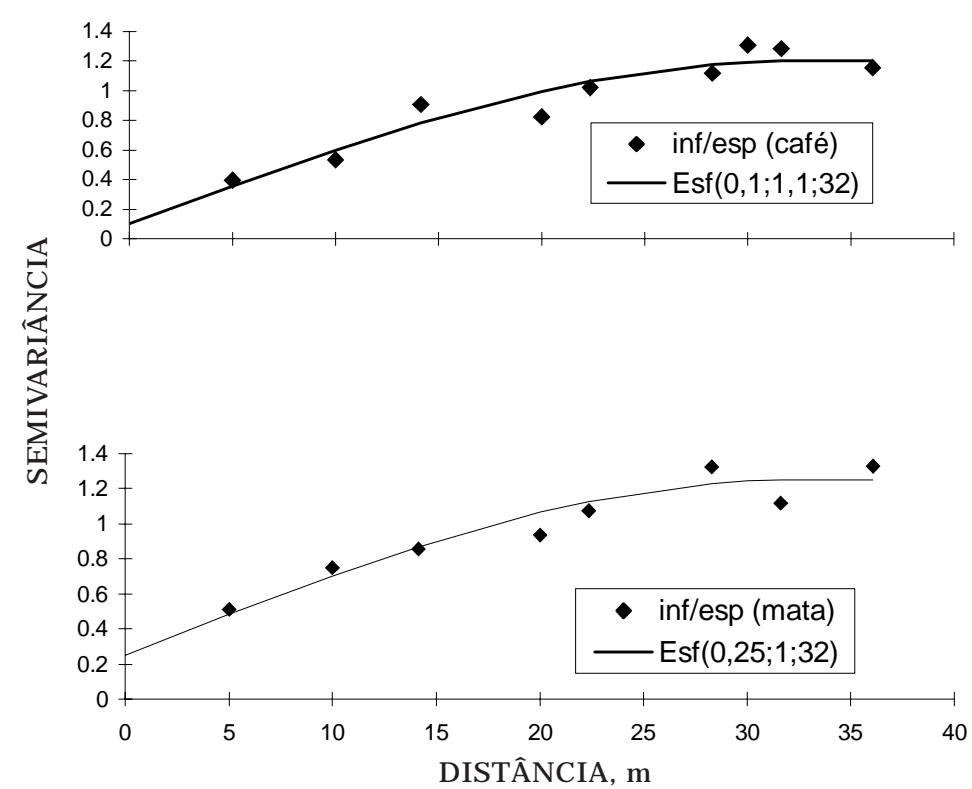

Figura 5. Semivariograma cruzado da infiltração de água no horizonte A e da sua espessura, nos cultivos de café e na mata/capoeira.

pepita puro (Figuras 3 e 4), denotando, assim, como observado para a infiltração, uma el evada irregularidade na variação deste atributo do solo ao longo do terreno.

O semivariograma cruzado mostrou correlação positiva entreinfiltração e espessura do horizonte $A$, no cultivo de café e na mata/capoeira, uma vez que seus valores encontraram-se acima da abscissa (Figura 5). Tal fato pode ser atribuído ao menor grau de degradação encontrado nessas áreas, uma vez que se observou a existência de uma cobertura vegetal controlada nas entrel inhas da cultura do café. Nas áreas sob pastagem, em que foi verificada uma condição ainda mais degradada, istoé, com presença de sulcos de erosão mais profundos, chegando, algumas vezes, a atingir o horizonte $\mathrm{Bt}$, não foi detectada correlação espacial da infiltração do horizonte A com a sua espessura. Portanto, em solos 
que apresentam el evado grau de degradação e, ou, uso intensivo, espera-se uma variação aleatória de seus atri butos (Berg \& Klamt, 1997; Bertolani, 1998), consi derando as al terações ocorridas no horizonte A, as quais se apresentam de forma bastanteirregular.

Em sol os degradados, que apresentam sulcos de erosão, como é o caso do sol o estudado, a dificuldade para detectar a dependência espacial podeser maior, quando comparada com solos sob manejos adequados, que propicia a manutenção do horizonte superficial. Corroborando esta afirmação, verificouse, em alguns tratamentos, a ausência de dependência espacial tanto para a infiltração, como para a espessura do horizonte A (Figuras 2, 3 e 4). Isto se deve não apenas aos fatores de formação do solo, que já imprimem uma variabilidade natural acentuada (Agbu \& Olson, 1990), mas principalmente às conseqüências dos processos erosivos e manejo incorreto dos solos (Kachanoski et al., 1985). Resultado semelhante foi encontrado por Berg \& Klamt (1997), estudando a variabilidade espacial de propriedades do solo. Os autores verificaram que a aplicação não-uniforme do calcário em diferentes quantidades levou a variações do $\mathrm{pH}$ a curtas distâncias, ocasionando o efeito pepita puro.

A formação de sulcos ocasionados peloescorrimento superficial da água provavelmente seja o fator de maior influência para a não-detecção da dependência espacial em sol os com evidentes si nais de degradação, como foi observado principal mente para a espessura do horizonte A (Quadro 2). N os locais em que são formados estes sulcos, tem-se a retirada de parte, ou mesmo de todo o horizonte superficial, alterando, conseqüentemente, de forma bastante irregular, os atributos do solo, e conferindo aos sol os degradados diferentes comportamentos em pequenas distâncias (em metros, ou até em centímetros).

Ressalta-se que, na área estudada, a utilização de menores distâncias entreamostras pode detectar dependência espacial para os atributos e condições em que foi detectado o efeito pepita puro. Isso indica que a dependência espacial dos atributos do solo é fortemente influenciada pela escala em que está sendo realizado o trabalho (Cambardel la et al., 1994), bem como pelo grau de degradação do solo.

\section{CONCLUSÕES}

1. A redução das taxas de infiltração deágua, do horizonte $\mathrm{E}$ para o $\mathrm{B}$ textural, em todos os cultivos analisados (café, pastagem e mata/capoeira), evidenciou o forte impedimento físico à percolação da água proporcionado pelo horizonte Bt.

2. A elevada amplitude dos valores de espessura do horizonte $A$ e o efeito pepita puro, verificado no cultivo de café e pastagem degradada, indicam a necessidade da manutenção da cobertura vegetal para evitar a remoção do horizonte superficial nesse solo.
3. A detecção da dependência espacial de atributos do sol o em áreas que apresentam avançado estádio de degradação é dificultada pela intensa atuação dos processos erosivos no horizonte superficial, os quais provocam variações bastante irregulares no horizonte superficial.

\section{LITE RATURA CITADA}

AGBU, P.A. \& OLSON, K.R. Spatial variability of soil properties in selected Illinois Mollisols. Soil Sci., 150:777-785, 1990.

ALBUQUERQUE, J.A.; REINERT, D.J . \& FIORIN, J.E. Variabilidade de solo e planta em Podzólico VermelhoAmarelo. R. Bras. Ci. Solo, 20:151-157, 1996.

BERG, M.V.D. \& KLAMT, E. Variabilidade espacial de características de solos na região do planalto médio, RS: I. Análise de variância por amostragem aninhada. R. Bras. Ci. Solo, 21:393-399, 1997.

BERTOLANI, F.C Caracterização física dos solos de uma microbacia hidrográfica, em Vera Cruz (SP). Campinas, Universidade de Campinas, 1998. 87p. (Tese de Mestrado)

BERTOLANI, F.C.; COELHO, M.R.; ROSSI, M.; MENK,J JR.F. \& PRADO, H. Levantamento pedológico semidetalhado do estado deSão Paulo: Fol ha de Marília. Memorial Descritivo. Campinas, I nstituto Agronômi co de Campinas, 2000a. 142p. (Serie Pesquisa APTA, Boletim Científico 01)

BERTOLANI, F.C.; PAZ GONZÁLEZ, A.; LADO LIÑARES, M.; VIDAL VÁZQUEZ, E. \& VIVAS MIRANDA, J.G. Variabilidade espacial da rugosidade superficial do solo calculada a partir de diferentes métodos. Bragantia, 59:227234, 2000b.

CAMBARDELLA, C.A.; MOORMAN, T.B.; NOVAK, J.M.; PARKIN, T.B.; KARLEN, D.L.; TURCO, R.F. \& KONOPKA, A.E. Field-scale variability of soil properties in Central I owa Soil. Soil Sci. Soc. Am. J ., 58:1501-1508, 1994

EMPRESA BRASILEIRA DE PESQUISA AGROPECUÁRIA EMBRAPA. Centro Nacional de Pesquisa de Solos. Sistema Brasileiro de Classificação de Solos. Rio de J aneiro, 1999.412p.

HILLEL, D. Applications of soil physics. New York, Academic Press, 1980. 385p.

INSTITUTO DE PESQUISA TECNOLÓGICA DO ESTADO DE SÃO PAULO - IPT. Orientação para o combate à erosão do Estado de São Paulo, Bacia do Peixe-Paranapanema. São Paulo, 1986. v.6. 739p. (IPT. Relatório, 24)

KACHANOSKI, R.G.; ROLSTON, D.E. \& J ONG, E. Spatial and spectral relationships of soil properties and microtopography I. Density and thickness of A horizon. Soil Sci. Soc. Am. J ., 49:804-811, 1985.

KECKLER, D. Surfer for windows. Version 6. Golden Software, Golden, CO. 1995.

KRIGE, D.G. A statistical approach to some basic mine valuation problems on the witwaterland. J. Chem. Metal. Min. Soc., 52:119-139, 1951. 
MCBRATNEY,A.B. \& WEBSTER, R. Choosing functions for semivariograms of soil properties and fitting them to sampling estimates. J. Soil Sci., 37:617-639, 1986.

PAZ, A.G. \& TABOADA, M.T. Medida del microrrelieve del suelo y estimación de la retención hídrica en depresiones de la superficie. J. Soc. Esp. Geom., 34:829-841, 1996.

PIMENTEL GOMES, F. Estatística moderna na pesquisa agropecuária. Piracicaba, POTAFOS, 1984. 160p.

PREVEDELLO, B.M.S. Variabilidade espacial de parâmetros de solo e planta. Piracicaba, Escola Superior de Agricultura "Luiz de Queiroz", 1987. 166p. (Tese de Doutorado)

REYNOLDS, W.D. \& ELRICK, D.E. Measurement of fieldsaturated hydraulic conductivity, sorptivity and conductivity-pressure head relationship using the "Guel ph permeameter". In: NATIONAL WATER WELL ASSOCIATION CONFERENCE ON CARACTERIZATION AND MONITORING OF THE VADOSE (Unsaturated) ZONE, Colorado, 1985. Proceedings. Denver, 1985. 25p.
SALVIANO, A.A.C. Variabilidade de atributos de solo e de Crotalaria juncea em solo degradado do município de Piracicaba-SP. Piracicaba, Escola Superior de Agricultura “Luiz de Queiroz", 1996. 91p. (Tese de Doutorado)

SNEDECOR, G.W. \& COCHRAN, W.G. Statistical methods. Ames, I owa State University Press, 1967. 583p.

SOUZA, L.S.; COGO, N.P. \& VIEIRA, S.R. Variabilidade de propriedades físicas equímicas do solo em um pomar cítrico. R. Bras. Ci. Solo, 21:367-372, 1997.

VAUCLIN, M.; VIEIRA, S.R.; VACHAUD, G. \& NIELSEN, D.R. The use of cokriging with limited field soil observation. Soil Sci. Soc. Am. J., 47:175-184, 1983.

VIEIRA, S.R.; HATFIELD, J .L.; NIELSEN, D.R. \& BIGGAR, J.W. Geostatistical theory and application to variability of some agronomical properties. Hilgardia, 51:1-75, 1983.

VIEIRA, S.R.; TILLOTSON, P.M.; BIGGAR, J.W. \& NIELSEN, D.R. Scaling of semivariograms and the kriging estimation of field-measured properties. R. Bras. Ci. Solo, 21: 525-533, 1997. 
